\title{
浅析质谱中的广义麦式重排
}

\author{
李鸿波 ${ }^{*}$, 王珀会 \\ 西南科技大学材料科学与工程学院, 四川绵阳 621010
}

摘要: 麦氏重排是对质谱分析中分子离子的重排反应提出的经验规则。对经典麦式重排的概念、裂解过程及其应用做进 一步拓展, 形成了广义麦式重排。在广义麦式重排中, $y-\mathrm{H}$ 的经典麦式重排是一步完成的六元环协同裂解, 分子离子亦 可通过六元环或五元环过渡态进行协同重排裂解, 发生相应的 $\gamma-R 、 \beta-H($ 或 R)的迁移, 产生不同的碎片离子。这种广 义麦氏重排在各种常见官能团化合物中均可发生, 其在质谱解析和化合物结构研究中具有广泛应用。

关键词: 广义麦氏重排; 协同裂解; 六元环; 五元环

中图分类号: G64; O6

\section{Analysis of General McLafferty Rearrangement in Mass Spectrometry}

\author{
Hongbo Li ${ }^{*}$, Pohui Wang
}

School of Materials Science and Engineering, Southwest University of Science and Technology, Mianyang 621010, Sichuan Province, P. R. China.

Abstract: McLafferty rearrangement is an empirical rule proposed for the rearrangement of molecular ions in mass spectrometry. Further expanding the concept, cleaving process and application of classic McLafferty rearrangement, the general McLafferty rearrangement is formed. In the general McLafferty rearrangement, the $\mathrm{\gamma}-\mathrm{H}$ transference of sixmembered ring cooperatively completes in one step. The cooperative rearrangement of molecular ions can also occur through a six-membered or five-membered ring transition state and the $\gamma-\mathrm{R}$ or $\beta-\mathrm{H}(\mathrm{R})$ of molecular ions accomplishes the transference, which results in different fragment ions. This general McLafferty rearrangement can occur in various compounds with most functional group, which is helpful in mass spectrometry analysis and compound structure research and extend the breadth of the applications.

Key Words: General McLafferty rearrangement; Cooperative cleavage; Six-membered ring;

Five-membered ring

麦氏重排(McLafferty rearrangement)是对质谱分析中分子离子的重排反应提出的经验规则, 于 1956 年由美国质谱学家麦克拉弗特(F. W. Mclafferty $)^{[1]}$ 提出。在经典麦式重排中, 当有机化合物含有 不饱和基团时(如 $\mathrm{C}=\mathrm{N} 、 \mathrm{C}=\mathrm{O} 、 \mathrm{C}=\mathrm{S}$ ), 与不饱和基团相连的 $\gamma-\mathrm{C}$ 上的 $\gamma-\mathrm{H}$ 会通过六元环过渡态过 渡转移到电离的原子上, 同时 $\alpha-\beta$ 键发生裂解, 产生新的碎片离子(图 1) ${ }^{[2,3]}$ 。

质谱检测中, 化合物会裂解生成离子、自由基和中性分子, 结合裂解产物的稳定性对经典麦式 重排做深入研究, 发现仍存在一些类似的其他重排裂解方式, 可称作广义麦式重排。在广义麦式重 排中, $\gamma-\mathrm{H}$ 的重排是一步完成的协同裂解, 因其符合有机化学反应中的协同重排机理, 该裂解方式

收稿：2019-05-06; 录用：2019-05-09; 网络发表：2019-06-03

“通讯作者, Email: li-honggg@163.com

基金资助: 四川省 2018-2020 年高等教育人才培养质量和教学改革项目(19sjjg15); 2019 年校级重点教育教学改革与研究项目(19xnzd15); 2018 年校级教学名师教改项目(18xnms01); 2015 年校级研究生精品课程建设项目(15jpk001) 
更合理(图 2)。此外, 广义麦式重排可以通过六元环或五元环过渡态进行多次协同重排裂解, 且重排 过程中能发生相应的 $\gamma-\mathrm{R} 、 \beta-\mathrm{H}$ (或 R) 迁移, 在诸多常见官能团化合物中均有应用, 有助于化学工作 者进行质谱分析和结构确证。

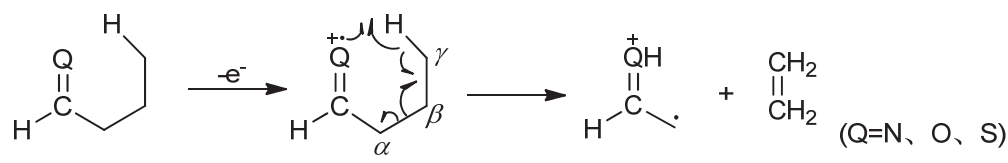

图 1 经典麦氏重排裂解过程

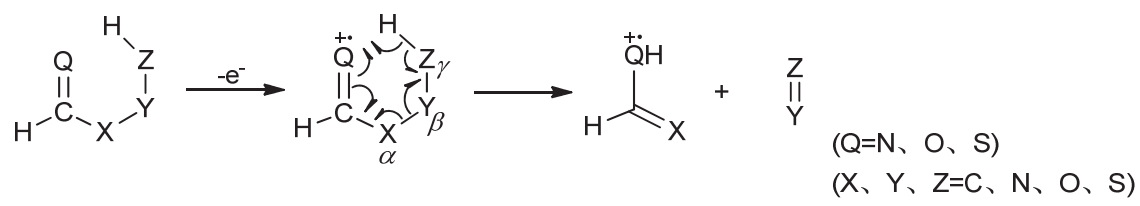

图 2 广义麦式重排的六元环协同裂解过程

\section{1 广义麦式重排}

\section{1 烷烃}

烷烃的 $\sigma$ 键半异裂生成分子离子，其 $\gamma-\mathrm{H}$ (或 $\mathrm{R}$ )通过六元环过渡态过渡转移到电离的碳原子上， 可以协同重排裂解为烯烃、小分子烷烃以及 $\mathrm{C}_{n} \mathrm{H}_{2 n}$ 的碎片离子(图 3)。此外, 烷烃分子离子的 $\beta$ - $\mathrm{H}$ (或 $\mathrm{R})$ 亦可通过五元环过渡态发生协同重排裂解, 生成烯烃、小分子烷烃以及卡宾或其衍生物碎片离子 (图 4)。

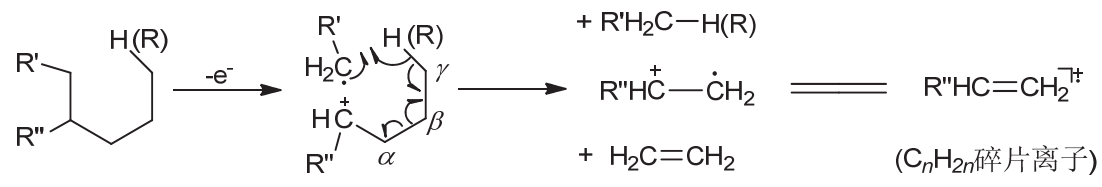

图 3 烷烃六元环麦式重排裂解

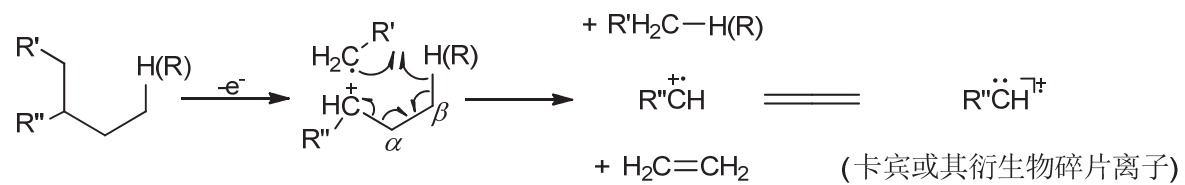

图 4 烷烃五元环麦式重排裂解

\section{2 烯烃}

烯烃的 $\pi$ 键容易半异裂生成分子离子，其 $\gamma-\mathrm{H}$ (或 $\mathrm{R}$ )可以通过六元环过渡态过渡转移到电离的 碳原子上, 同时 $\alpha-\beta$ 键均裂产生小分子烯烃和 $\mathrm{C}_{n} \mathrm{H}_{2 n}$ 的碎片离子(图 5)。此外, 烯烃分子离子的 $\beta$ $\mathrm{H}$ (或 $\mathrm{R}$ )亦能经过五元环过渡态过渡转移到电离的碳原子上, 协同重排裂解为小分子烯烃和卡宾衍 生物碎片离子(图 6)。

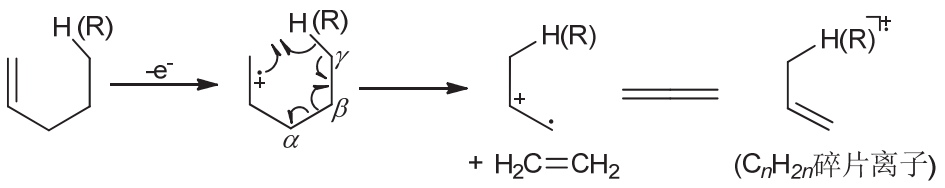

图 5 烯烃六元环麦式重排裂解 


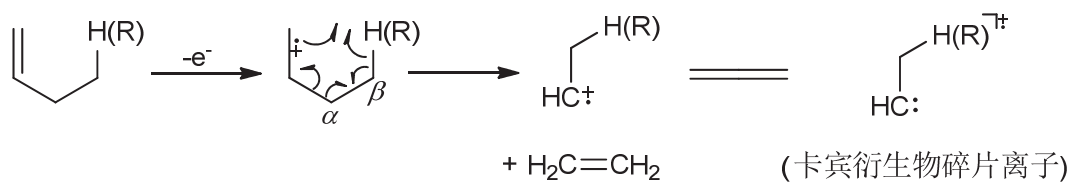

图 6 烯烃五元环麦式重排裂解

\section{3 炔烃}

炔烃的 $\pi$ 键容易半异裂生成分子离子, 其 $\gamma-\mathrm{H}$ (或 R) 可以通过六元环过渡态过渡转移到电离的 碳原子上, 可能协同重排裂解为烯烃分子和丙二烯型碎片离子(图 7)。炔烃经五元环过渡态发生裂解 时，生成的碎片离子十分不稳定，故发生裂解的概率小，即使能发生，其丰度也很低。

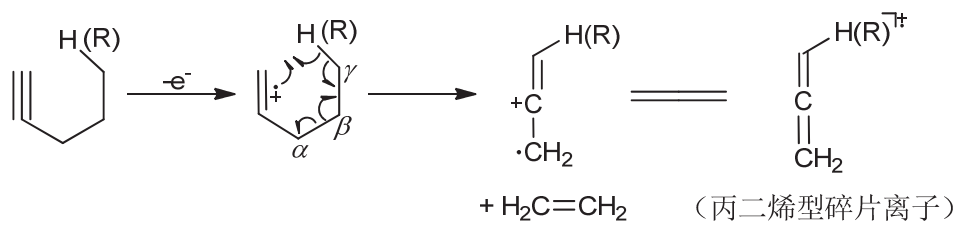

图 7 炔烃六元环麦式重排裂解

\section{4 芳烃}

经典麦式重排中, 芳烃 $\gamma-\mathrm{H}$ 经六元环过渡态过渡转移到电离的碳原子上, 会在质谱图中呈现特 征碎片离子峰, 例如正丙基苯可产生 $m / z 92$ 的碎片离子峰 ${ }^{[3]}$ 。广义麦式重排中, 芳烃 $\gamma-\mathrm{H}$ (或 R)均能 发生六元环的协同重排裂解(图 8)。而芳烃 $\beta$ - $\mathrm{H}$ (或 R)经五元环协同裂解, 产生极不稳定的环状卡宾 衍生物碎片离子，故其裂解概率小，碎片离子丰度低，甚至不发生。

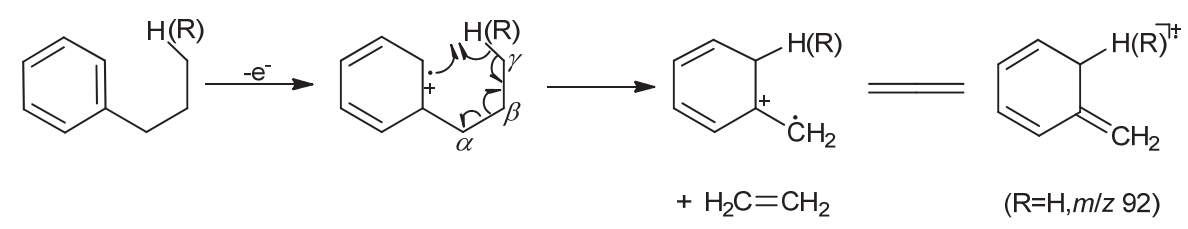

图 8 芳烃六元环麦式重排裂解

\section{5 卤代烃}

卤素基团 $(\mathrm{X})$ 的 $n$ 电子易裂解生成分子离子，其 $\gamma-\mathrm{H}$ (或 $\mathrm{R}$ )可经六元环过渡态重排裂解, 最终生 成烯烃、卤化氢(或卤代烃)及 $\mathrm{C}_{n} \mathrm{H}_{2 n}$ 的碎片离子(图 9)。其 $\beta-\mathrm{H}$ (或 $\mathrm{R}$ )亦能经五元环过渡态重排裂解, 且具有两种不同的裂解方式，可生成多种碎片离子(图 10)。

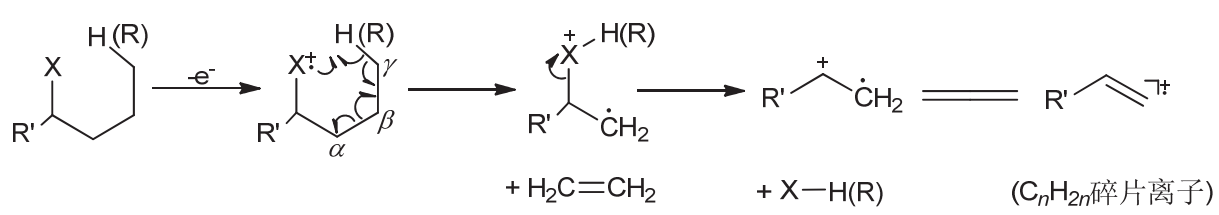

图 9 卤代烃六元环麦式重排裂解

\section{6 醇、酚和醚}

醇和醚的氧原子经 $n$ 电子裂解生成分子离子，其 $\gamma-\mathrm{H}$ (或 R) 可经六元环过渡态重排裂解，最终 生成烯烃、 $\mathrm{H}_{2} \mathrm{O}$ (醇或醚)及 $\mathrm{C}_{n} \mathrm{H}_{2 n}$ 的碎片离子(图 11); 其 $\beta-\mathrm{H}$ (或 R)亦可经五元环过渡态通过两种不 同的裂解方式协同重排，生成多种碎片离子(图 12)。 


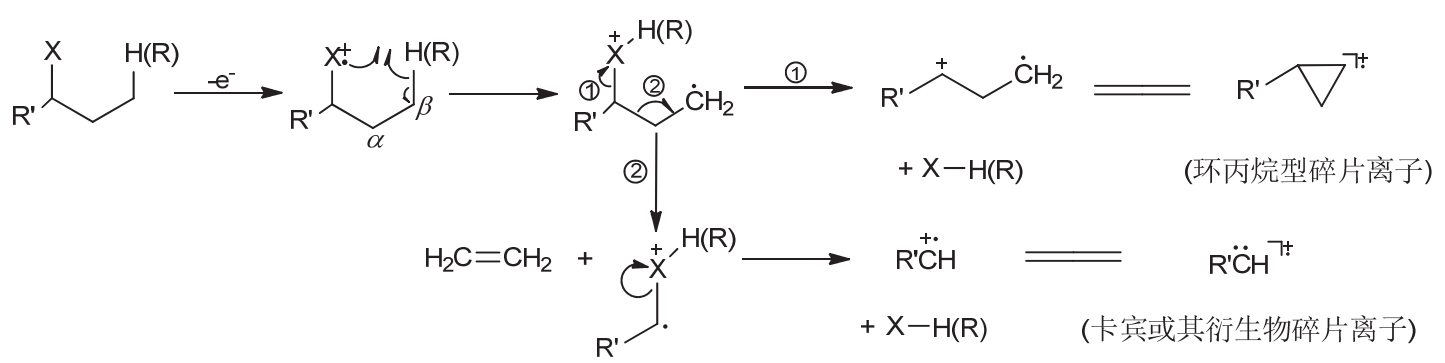

图 10 卤代烃五元环麦式重排裂解

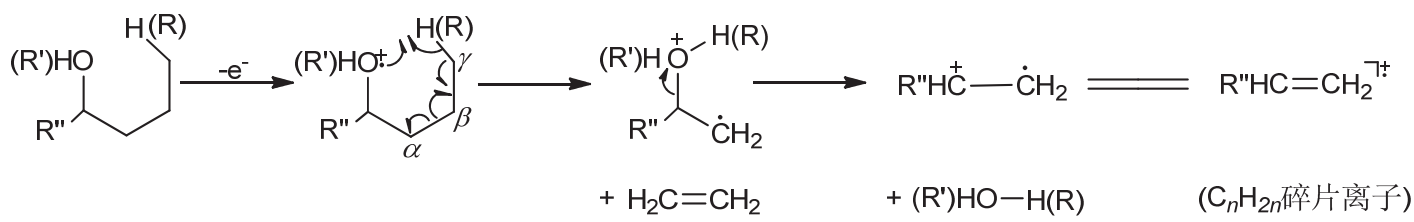

图 11 醇和醚六元环麦式重排裂解

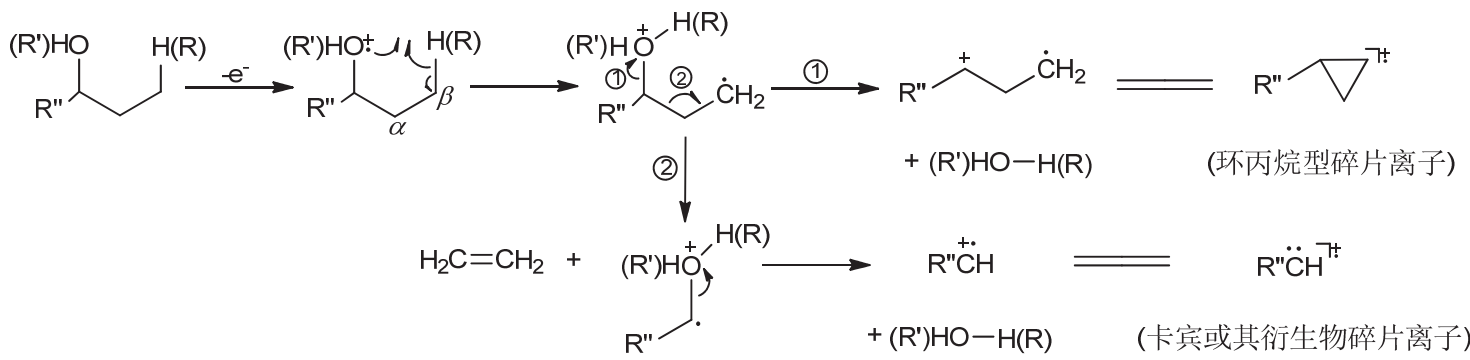

图 12 醇和醚五元环麦式重排裂解

酚及其醚的 $\gamma-\mathrm{H}$ (或 R) 可经六元环过渡态重排裂解, 最终生成烯烃、 $\mathrm{H}_{2} \mathrm{O}$ (醇或醚)及苯炔分子离 子(图 13); 其 $\beta-\mathrm{H}$ (或 $\mathrm{R}$ )经五元环过渡态通过两种不同方式进行重排裂解, 均生成苯并环丙烷分子 离子(图 14)。

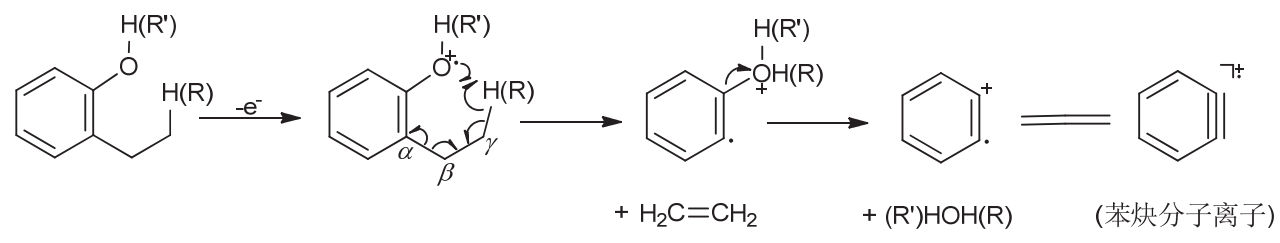

图 13 酚及其醚六元环麦式重排裂解

\section{7 腈类化合物}

腈类化合物 $n$ 电子裂解或 $\pi$ 键半异裂生成相应的分子离子，其 $\gamma-\mathrm{H}$ (或 R)均可经六元环过渡态 协同重排裂解，产生乙烯亚胺型分子离子(图 15), 此裂解过程与炔烃化合物相似。同时, 腈类化合 物经五元环过渡态进行协同重排裂解的概率小，即使能发生，碎片离子的丰度也很低。

\section{8 胺类化合物}

与醇和醚相似, 胺类化合物中氮原子的 $n$ 电子发生裂解生成分子离子, 其 $\gamma-\mathrm{H}($ 或 $\mathrm{R}$ )可经六元 环过渡态重排裂解, 最终生成烯烃、小分子胺及 $\mathrm{C}_{n} \mathrm{H}_{2 n}$ 的碎片离子(图 16); 其 $\beta-\mathrm{H}$ (或 $\mathrm{R}$ )亦可经五元 环过渡态按两种不同方式进行重排裂解，生成多种碎片离子(图 17)。 


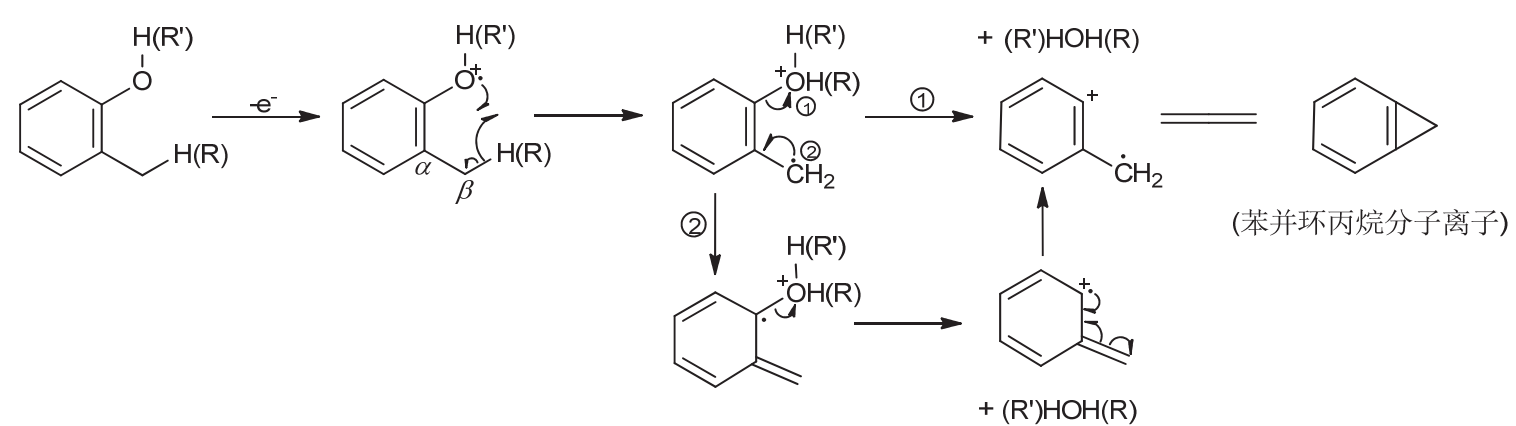

图 14 酚及其醚五元环麦式重排裂解

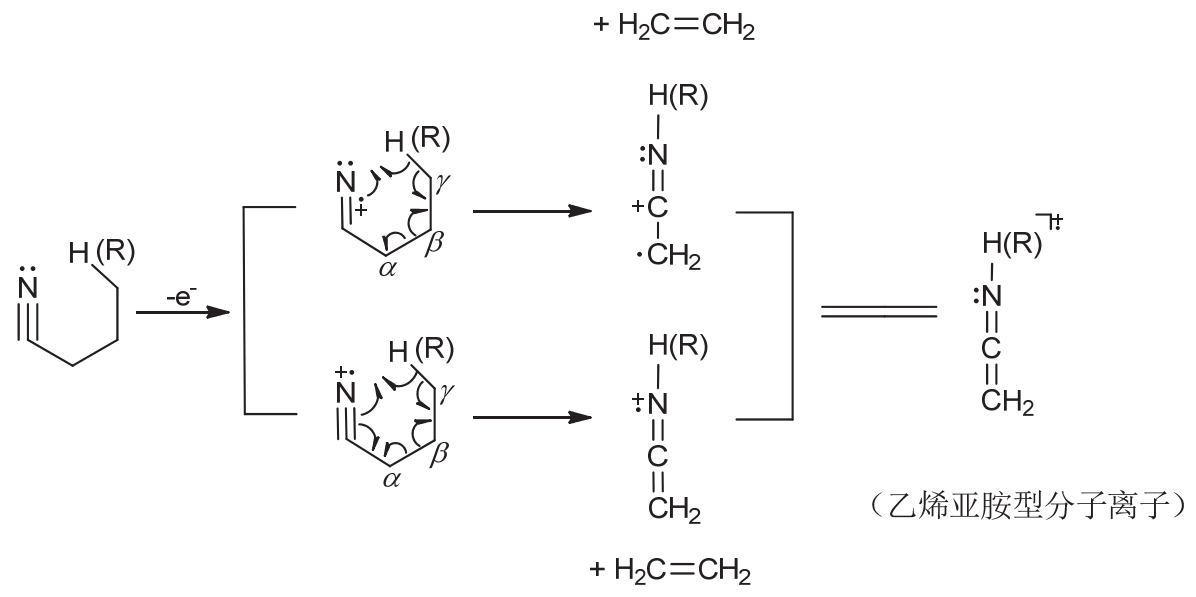

图 15 腈类化合物六元环麦式重排裂解

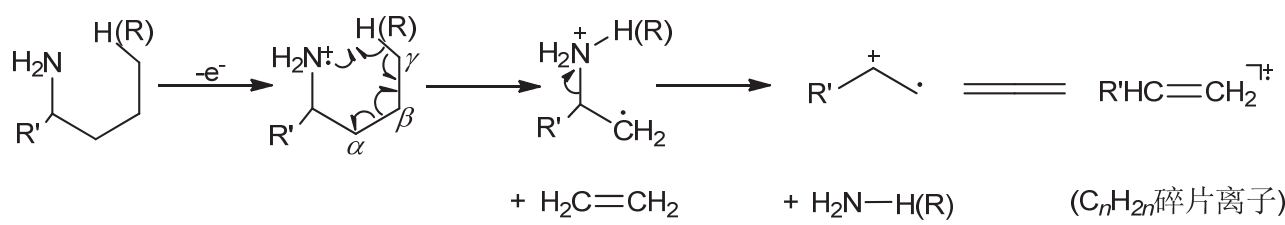

图 16 胺类化合物六元环麦式重排裂解

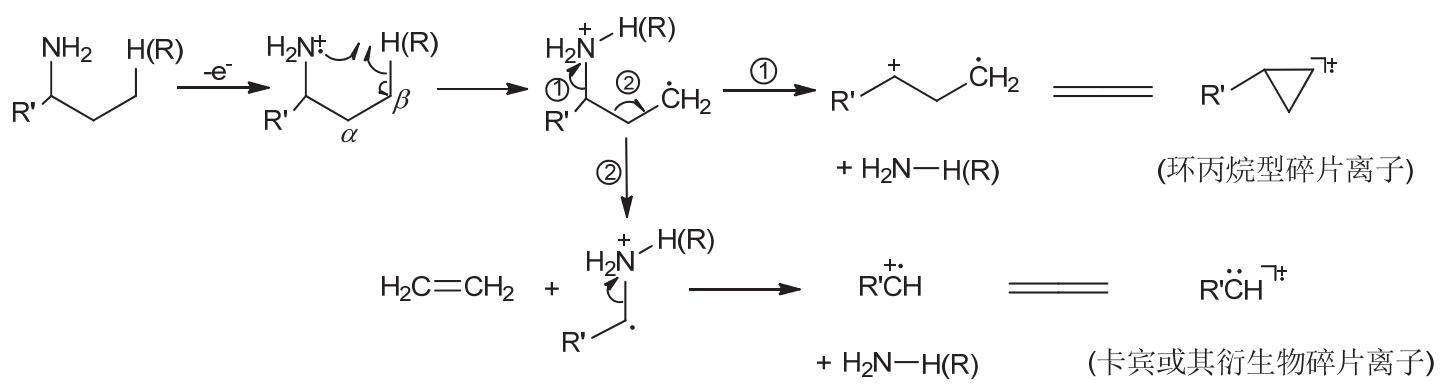

图 17 胺类化合物五元环麦式重排裂解

\section{9 杂环化合物}

杂环化合物 $\pi$ 键半异裂生成分子离子, 其 $\gamma-\mathrm{H}$ (或 R) 能发生协同重排裂解(图 18), 此裂解过程与 芳烃类似。若经五元环过渡态协同裂解，生成的碎片离子极不稳定，发生的概率小甚至不发生。 


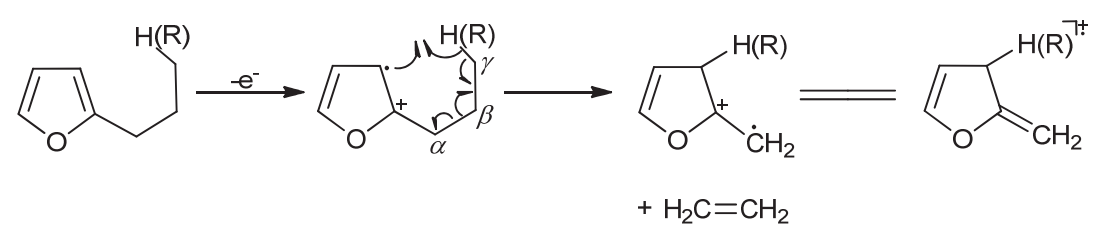

图 18 杂环化合物六元环麦式重排裂解

\subsection{0 酫和酮}

醛和酮是发生经典麦式重排的常见化合物, 若重排裂解后的碎片离子仍具有进行麦式重排的条 件, 即含有不饱和基团(羰基 $\mathrm{C}=\mathrm{O}$ )和 $\gamma-\mathrm{H}$, 其可进一步发生麦式重排, 甚至连续发生多次麦式重排。 以 2,2-二乙基丁醛为例, 其可发生连续 3 次麦式重排, 最终裂解为乙醛分子离子(图 19)。由此, 醛 可能发生 3 次麦式重排, 而酮则可能会发生 6 次麦式重排。

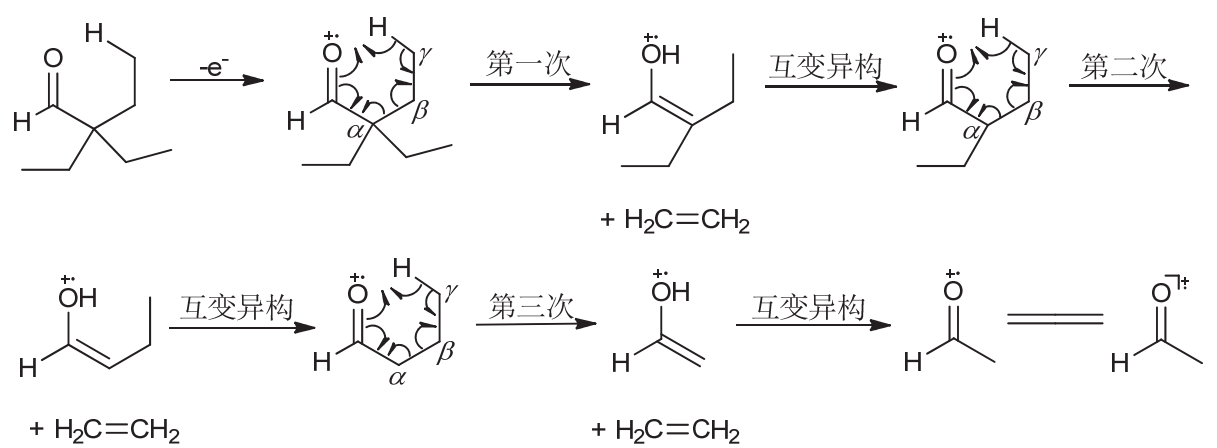

图 19 2,2-二乙基丁醛麦氏重排裂解

值得注意的是, 醛和酮在麦氏重排裂解过程中, 也有可能发生 $\gamma$-R 的迁移产生乙烯基烷基醚型 碎片离子, 若其结构中仍有 $\gamma-\mathrm{H}$ (或 R), 可进一步发生醚类的广义麦氏重排。例如, 2-正丙基戊醛可 以先经 $\gamma-\mathrm{CH}_{3}$ 迁移, 再发生 $\gamma-\mathrm{CH}_{3}$ (1)式)或 $\gamma-\mathrm{H}$ (2)式)迁移, 最终裂解成的碎片离子均为乙炔分子离 子(图 20); 而 5-壬酮先 $\gamma-\mathrm{CH}_{3}$ 迁移、再 $\gamma-\mathrm{H}$ (或 $\gamma-\mathrm{CH}_{3}$ ) 迁移, 最终都裂解为丙二烯分子离子(图 21)。

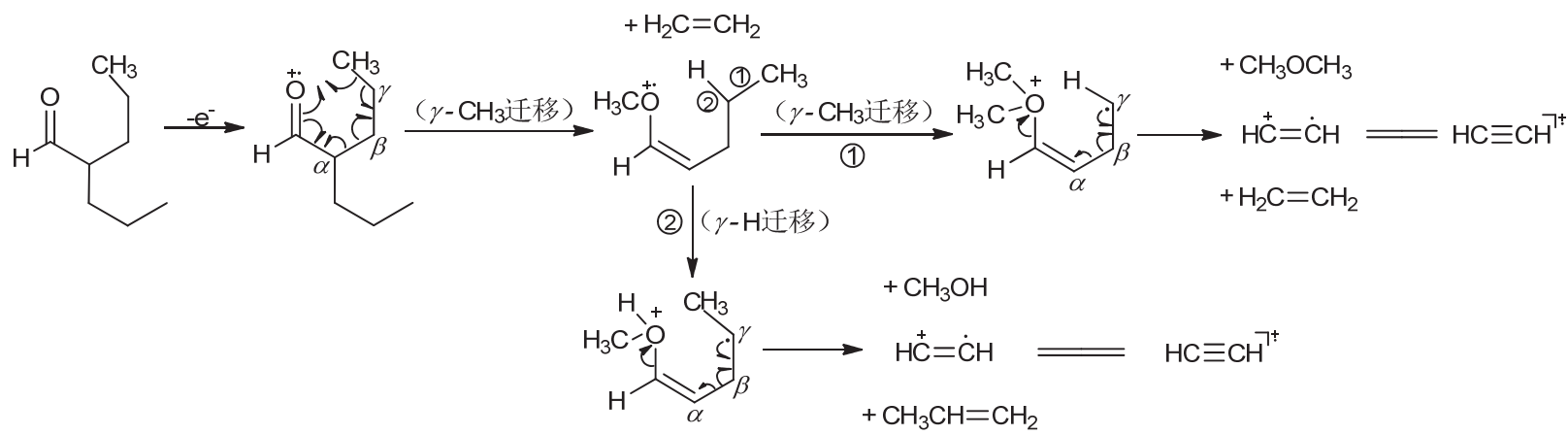

图 20 2-正丙基戊醛广义麦氏重排裂解

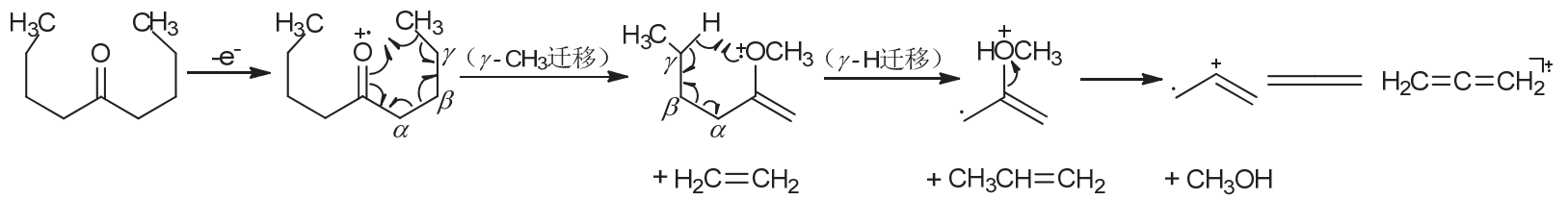

图 21 5-壬酮广义麦氏重排裂解 


\subsection{1 羧酸及其衍生物}

与醛和酮类似, 广义麦式重排中羧酸及其衍生物均能发生多次麦式重排。例如羧酸和酰卤可以 发生 3 次麦式重排, 酯可以发生 4 次麦式重排, 酰胺甚至可以发生 5 次麦式重排, 而酸酐由于有两 个羰基, 其发生麦式重排的次数会更多, 裂解过程更为复杂。以 2,2-二乙基丁酸乙酯为例, 连续经 4 次麦式重排, 最终裂解为乙酸分子离子(图 22)。

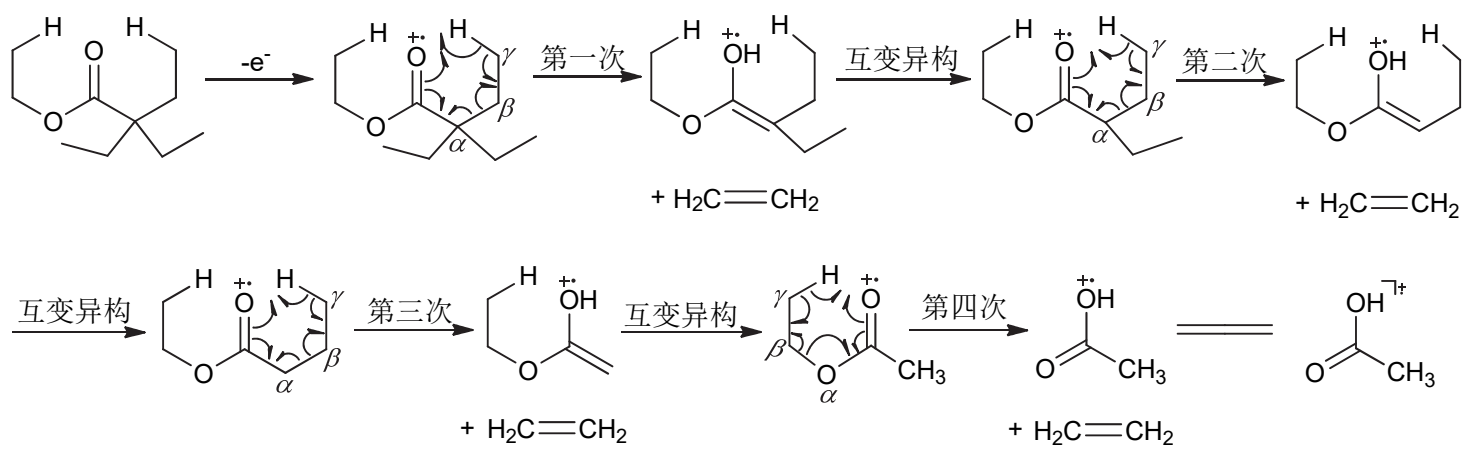

图 22 酯类化合物多次麦氏重排裂解

羧酸及其衍生物也可以发生 $\gamma-\mathrm{R}$ 迁移的广义麦氏重排, 产生的碎片离子可能继续进行醚类的广 义麦氏重排，产生新的碎片离子(图 23，图 24)。

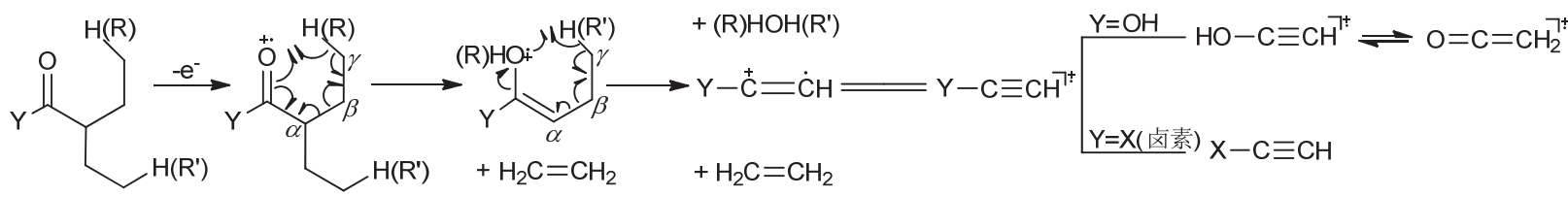
$\mathrm{Y}=\mathrm{OH}, \mathrm{X}($ 卤素 $)$

图 23 羧酸和酰卤的广义麦氏重排裂解

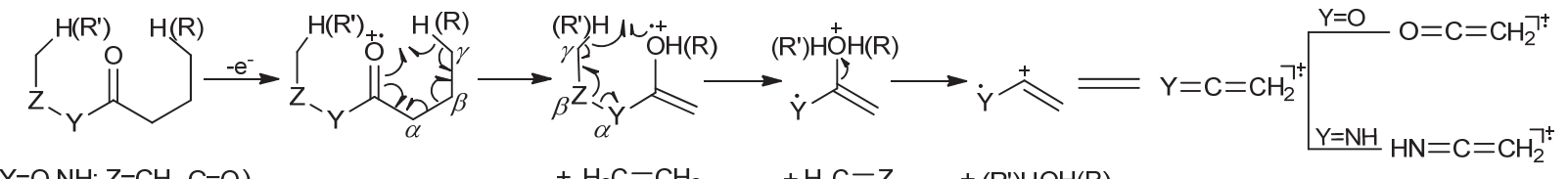
$\left(\mathrm{Y}=\mathrm{O}, \mathrm{NH} ; \mathrm{Z}=\mathrm{CH}_{2}, \mathrm{C}=\mathrm{O}\right)$

图 24 酸䣶、酯和酰(亚)胺的广义麦氏重排裂解

\section{2 结语}

麦式重排是质谱分析中十分常见且重要的经验规则, 其可以解释有机化合物质谱中系列碎片离 子的裂解方式与过程, 从而有效确证有机化合物结构。广义麦氏重排中, 经典麦式重排是经过六元 环过渡态发生的协同裂解; 此外, 有机化合物也可以通过六元环或五元环过渡态进行协同裂解，发 生 $\gamma-\mathrm{H}$ (或 R)、 $\beta-\mathrm{H}$ (或 R) 的迁移, 产生更多的碎片离子, 裂解过程合理可行, 便于质谱解析。广义 麦式重排是在经典麦式重排基础上的进一步拓展, 其裂解方式与规律有助于质谱解析、化合物结构 研究及有机化学反应机理的深入理解。

[1] Mclafferty, F. W. 质谱解析. 王光辉, 译. 北京: 化学工业出版社, 1987.

[2] 范康年, 陆靖. 谱学导论. 北京: 高等教育出版社, 2011: 204-205.

[3] 邓芹英, 刘岗, 邓慧敏. 波谱分析教程. 北京: 科学出版社, 2007: 232-239. 